\title{
THE PH.ASE STABILITY OF MICROPULSES FROM PSR 1133+16
}

\author{
V. D. Pugachev, Yu. P. Shitov, and A. G. Soin \\ Radio Astronomy Department, Lebedev Physical Institute
}

\section{Introduction}

As is very well known, there are two different kinds of time structure of the individual pulses of pulsars. There are microstructures with time scales less than $1 \mathrm{~ms}$ and subpulse structures, the usual scale of which is of the order of $10 \mathrm{~ms}$. Subpulses are a result of the radiation beam rotation and in many

\section{Table 1}

\begin{tabular}{|l|rr|}
\hline Frequency (MHz) & $102.5^{a}$ & 40.0 \\
\cline { 2 - 3 } Number of frequency channels & 8 & 16 \\
Sampling interval (ms) & 0.70 & 1.41 \\
Dispersion broadening (ms) & 0.2 & 3.1 \\
Time constant (ms) & 1.0 & 1.0 \\
Number of pulses & 200 & 500 \\
\hline \multicolumn{2}{|c|}{${ }^{a}(103.7$ and $101.3 \mathrm{MHz})$}
\end{tabular}

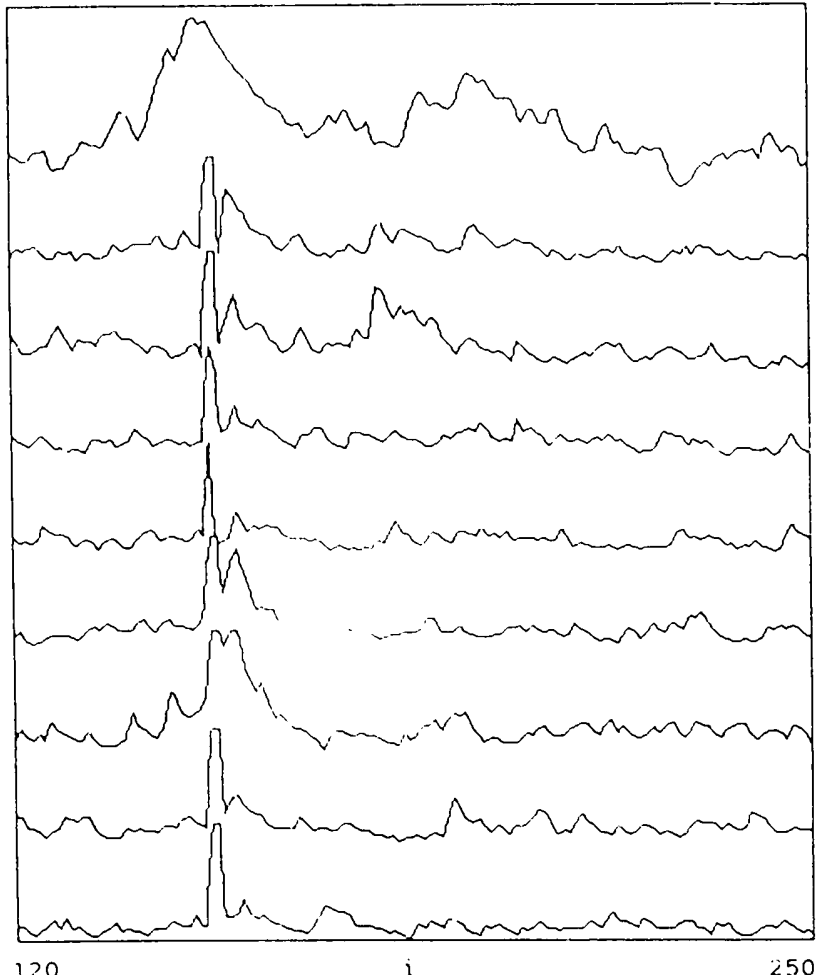

Figure 1 Data recorded from PSR $1133+16$ on 17 November 1986 near $102.5 \mathrm{MHz}$. The top trace is the average profile of 200 periods. The next four traces are four data channels at $103.719,103.714,103.789$, and $103.784 \mathrm{MHz}$. The next four traces are of the same channels but for the next sequential pulse period. cases demonstrate some regularity of arrival phases. In contrast, microstructure looks rather like a short pulse of noise with random phases of micropulse appearance in the radio emission window of the pulsar. Because the appearance phases of micropulses are random in the pulsar period, it is very difficult to establish what the fundamental character of these micropulses is: are they a temporal variation of radio emission intensity or the result of narrow beam rotation? The shortest micropulses with scales of the order of 1-10 $\mu \mathrm{s}$ (for ordinary long periodic pulsars) are apparently temporal variations, but the question is quite open for scales $\geq 100 \mu \mathrm{s}$.

We report here an unusual result from the investigation of individual pulses of PSR $1133+16$ at meter wave lengths. We find that some micropulses (duration $<700 \mu \mathrm{s}$ ) maintain exactly the same phase during several pulsar periods.

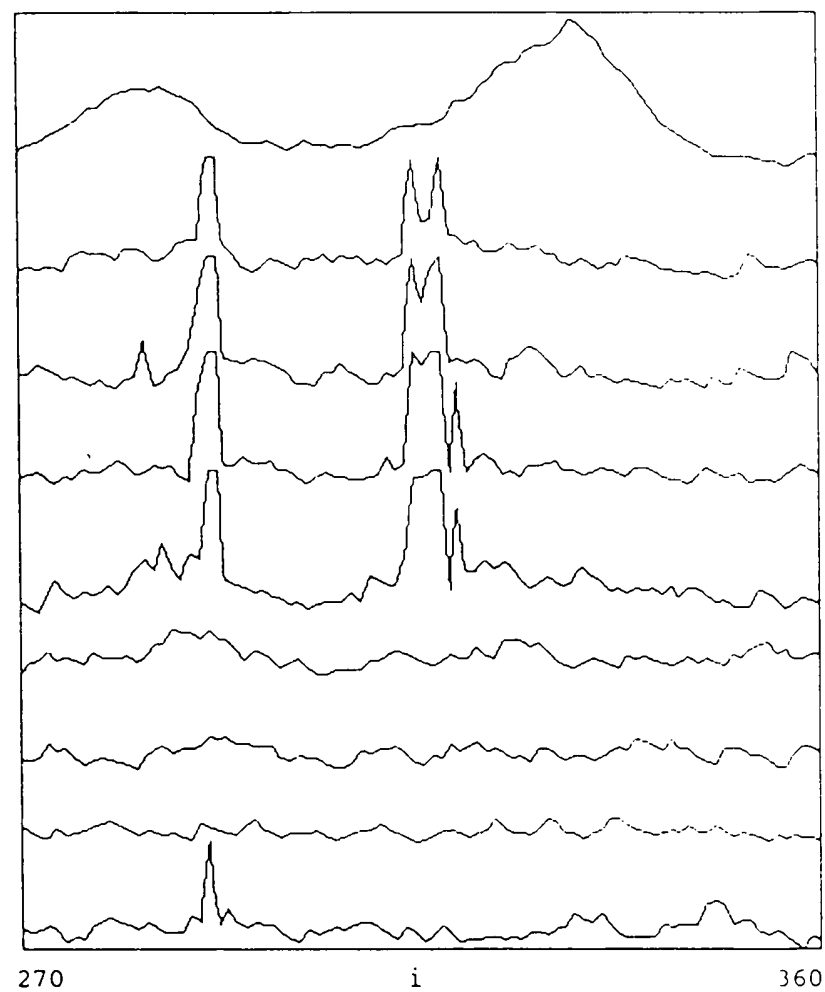

Figure 2 Data recorded from PSR 1133+16 on 17 November 1986 near $102.5 \mathrm{MHz}$. The top trace is the average profile of 200 periods. The next four traces are four data channels at $101.300,101.295,101.290$, and $101.285 \mathrm{M} \mathrm{Hz}$. The next four traces are of the same channels but for the next sequential pulse period. 


\section{Observations}

The observations of PSR $1133+16$ were made with two radio telescopes: the large cophased antenna (BSA) at $102.5 \mathrm{MHz}$ and the east-west arm of the cross radio telescope (DKR-1000) at $40 \mathrm{MHz}$. The individual pulses were recorded onto magnetic tape using a multichannel $(32 \times 5-\mathrm{kHz})$ radiometer. The time resolution was limited by dispersion broadening $\tau_{\mathrm{DM}}$ within the $5-\mathrm{kHz}$ bandwidth channel and by the rate of the analog-to-digital converter in the recording system, which was $70 \mu$ s per channel. The main observation parameters are shown in table 1.

The first observations of PSR $1133+16$ were made in November 1986 at two separate frequencies around $102.5 \mathrm{MHz}( \pm 1.2 \mathrm{MHz})$ to study the frequency correlation of subpulse shapes. Many strong and very short subpulse details were recorded with durations less than the sampling interval $(700 \mu \mathrm{s})$, which do not as a rule correlate at separate frequencies.

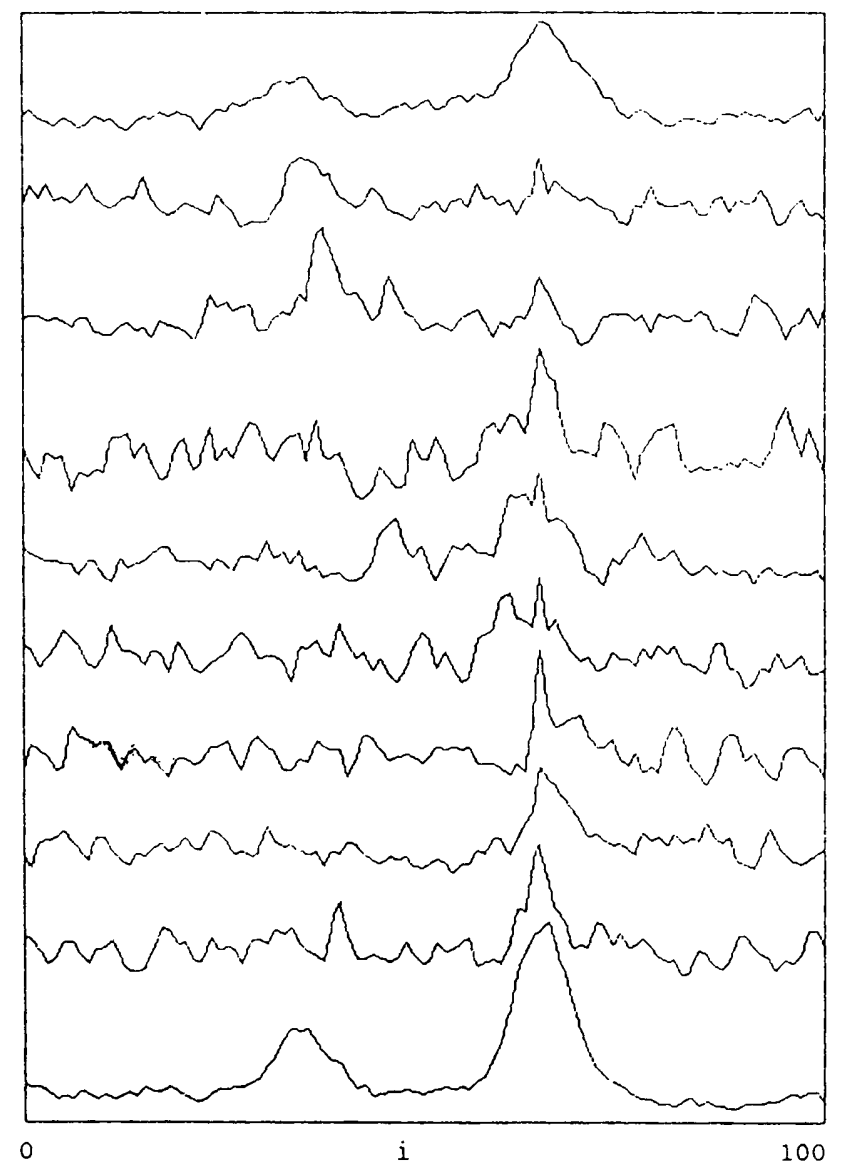

Figure 3 Data recorded from PSR $1133+16$ on 22 December 1988 near $40 \mathrm{MHz}$. The top trace is the average profile of 500 periods. The pulse numbers for the next eight traces are, respectively, $25,71,85,85,471,472,472$, and 476. The frequencies of these channels are, in the same order, 40.015, 39.97, 40.015, 39.97, 40.01, 39.995, 39.975, and $39.955 \mathrm{M} \mathrm{Hz}$. The bottom trace is the average profile of the 31 pulses with $S / N \geq 6$.
However, at the same time it was noticed that some strong, short micropulses repeat after a number of periods at exactly the same arrival phase (accuracy $\geq 300 \mu \mathrm{s}$ ) in the same single frequency channei. Only a few strong examples of such micropulses can take place during one scan of observation (200 periods). And what is more, as analysis shows, micropulses remember some particular phases after a few days.

Figures 1 and 2 display examples of this effect at $102.5 \mathrm{MHz}$. To check this effect a new 14-day set of observations at $102.5 \mathrm{MHz}$ was made in August 1987. A distinct phase stability effect was detected in 4 days of this set. As statistical analysis shows, only $\approx 1 \%$ of the individual puises of PSR $1133+16$ show phase stable micropulses at $102 \mathrm{MHz}$ (in our case of 8 frequency channels).

In December 1988 observations at $40 \mathrm{MHz}$ were made to study this effect at low frequencies. At this frequency the sensitivity of the radio telescope allowed us to record in.one scan of observations a few dozen pulses with signal-to-noise ratio, $S / N \geq 6$ at the pulse peak. We detected many pulses which have short and quite strong details. As at $102 \mathrm{MHz}$ the shortest pulse details have a duration less than or equal to the sampling interval and some of them demonstrate the phase stability effect. Figures 3 and 4 display examples of this effect at $40 \mathrm{MHz}$ in profile components I and II, respectively.

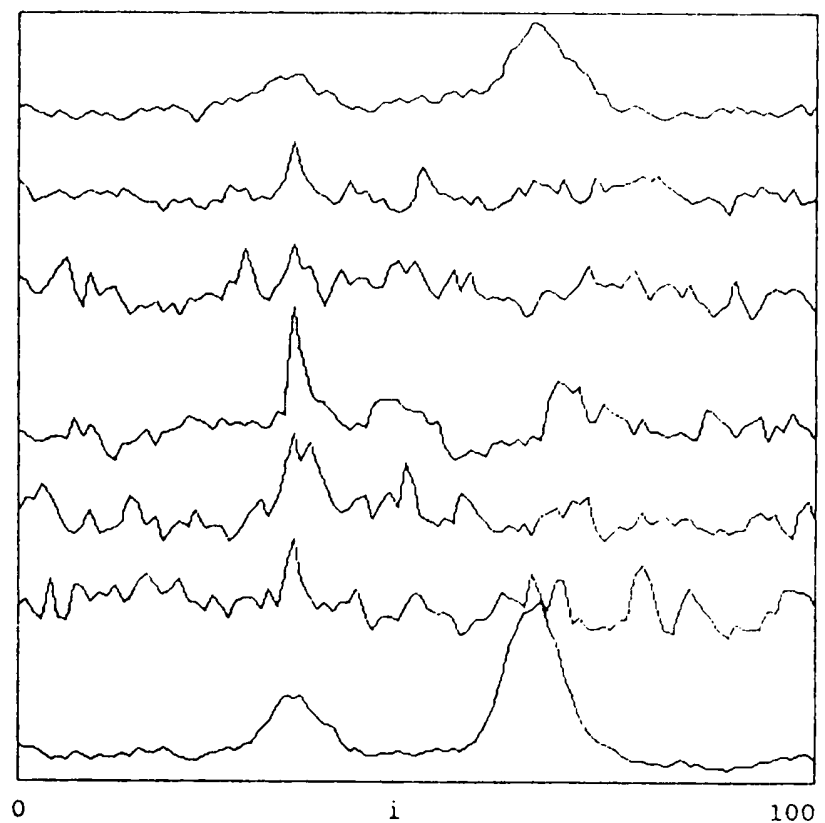

Figure 4 Data recorded from PSR 1133+16 on 22 December 1988 near $40 \mathrm{MHz}$. The top trace is the average profile of 500 periods. The pulse numbers for the next five traces are, respectively, $13,20,20,20$, and 87 . The frequencies of these channels are, in the same order, 39.955, $39.995,39.990,39.965$ and $39.995 \mathrm{MHz}$. The bottom trace is the average profile of the 31 pulses with $S / N \geq 6$. 


\section{Conclusions}

The detection of phase stable micropulses in PSR 1133+16 leads to important conclusions:

1. It means that micropulses with scales of the order of $500 \mu \mathrm{s}$, or at least some of them, are the result of the rotation of a a narrow radio emission beam, and not a result of time variations in intensity.
2. The existence of emission with such narrow beams-i.e. with widths $\delta \theta \leq 0.15^{\circ}$ in the polar cap model of the pulsar-leads to a direct estimate of the Lorentz factor of the emitting particles $\gamma=$ $360 /(2 \pi \delta \theta) \geq 380$.

3. Phase stability during a large number of periods shows the existence of long lived, compact, and isolated regions of radio emission in the pulsar magnetosphere. 